$6-2006$

\title{
Escape of Trajectories From a Vase-Shaped Cavity
}

\author{
Paul Hansen \\ William \& Mary \\ Kevin A. Mitchell \\ John B. Delos \\ William \& Mary, jbdelos@wm.edu
}

Follow this and additional works at: https://scholarworks.wm.edu/aspubs

Part of the Physics Commons

\section{Recommended Citation}

Hansen, Paul; Mitchell, Kevin A.; and Delos, John B., Escape of Trajectories From a Vase-Shaped Cavity (2006). Physical Review E, 73(6).

https://doi.org/10.1103/PhysRevE.73.066226

This Article is brought to you for free and open access by the Arts and Sciences at W\&M ScholarWorks. It has been accepted for inclusion in Arts \& Sciences Articles by an authorized administrator of W\&M ScholarWorks. For more information, please contact scholarworks@wm.edu. 


\title{
Escape of trajectories from a vase-shaped cavity
}

\author{
Paul Hansen, ${ }^{1, *}$ Kevin A. Mitchell, ${ }^{2, \dagger}$ and John B. Delos ${ }^{1, \hbar}$ \\ ${ }^{1}$ Department of Physics, College of William and Mary, Williamsburg, Virginia 23187-8795, USA \\ ${ }^{2}$ School of Natural Sciences, University of California, Merced, California 95344, USA
}

(Received 26 January 2006; published 28 June 2006)

\begin{abstract}
We consider the escape of ballistic trajectories from an open, vase-shaped cavity. Such a system serves as a model for microwaves escaping from a cavity or electrons escaping from a microjunction. Fixing the initial position of a particle and recording its escape time as a function of the initial launch direction, the resulting escape-time plot shows "epistrophic fractal" structure-repeated structure within structure at all levels of resolution with new features appearing in the fractal at longer time scales. By launching trajectories simultaneously in all directions (modeling an outgoing wave), a detector placed outside the cavity would measure a train of escaping pulses. We connect the structure of this chaotic pulse train with the fractal structure of the escape-time plot.
\end{abstract}

DOI: 10.1103/PhysRevE.73.066226

PACS number(s): 05.45.Ac, 05.45.Df, 42.60.Da, 32.80.Rm

\section{INTRODUCTION}

We study a billiard system that exhibits "chaotic escape," in which the amount of time to exit the system depends sensitively on initial conditions. Such systems display fractal structure-a hallmark of chaotic dynamics. Well known fractals may show regular self-similarity (e.g., the Cantor set), asymptotic self-similarity (e.g., sequences of perioddoubling bifurcations) or statistical self-similarity (e.g., coastlines and clouds). Certain chaotic systems may exhibit a type of fractal structure called "epistrophic" self-similarity $[1,2]$. (The word "epistrophe" is a term from rhetoric, meaning a regular ending following a variable beginning.) An epistrophic fractal may have self-similar structures as well as variable structures at all levels of resolution. Previously, we studied such fractals in a model two-dimensional map $[1,2]$ and in the ionization of hydrogen in parallel electric and magnetic fields $[3,4]$. In those cases, the variable structures came to dominate as we went deeper into the fractal.

This paper describes a new example of epistrophic fractal structure: the escape of trajectories (e.g., light, microwaves, or electrons) from a perfectly reflecting open vase-shaped cavity [5]. The fractal structure is analyzed using the topological methods developed in our previous work.

Miller [6] may have been the first to compute and identify chaotic scattering trajectories, which he called "chattering" trajectories, in atom-molecule collisions. Later, in the study of He colliding with $\mathrm{I}_{2}$, Davis [7] and Tiyapan and Jaffé [8] gave evidence of asymptotic self-similarity in plots of final action versus initial phase. For more background on chaotic scattering see the book by Gaspard [9]. Motivated by this earlier work, we found analogous phenomena in the escape of high-Rydberg electrons from hydrogen atoms in parallel electric and magnetic fields $[3,4]$. Treating the electron motion classically, we presented graphs of the escape time of

\footnotetext{
*Electronic address: pch@stanford.edu

†Electronic address: kmitchell@ucmerced.edu

†Electronic address: jbdelo@wm.edu
}

the electron (the time between the excitation of the electron and its arrival at a detector) versus the initial launch angle of the electron. These escape-time plots exhibit intervals over which the escape time is a smooth function of launch angle, but for which the escape time goes to infinity at each endpoint. These intervals of continuous escape time are called escape segments. The set of escape segments displays what we called epistrophic self-similarity, with asymptotically self-similar sequences of escape segments (called "epistrophes") as well as "unexpected" escape segments (called "strophes") occurring at all levels of resolution and at arbitrarily long times.

The purpose of this paper is to show that similar epistrophic fractal structure occurs in escape of light or particles from the vase cavity. In so doing, we provide a new physical system in which the effects of epistrophic fractals may be observed experimentally [10].

In Refs. [2,11], we developed a new form of symbolic dynamics that establishes the existence and structure of a minimal required set of escape segments. This symbolic dynamics, which we call homotopic lobe dynamics, is used here to interpret the fractal structure of the escape-time plot for the vase cavity. Homotopic lobe dynamics belongs to a larger field of research that applies symbolic techniques to the study of chaotic transport, including escape and scattering processes. We have been particularly influenced by the mathematical work of Jung and collaborators [12-16], Easton $[17,18]$, Rom-Kedar $[19,20]$, and more recently by the work of Collins [21].

We mention other recent work on chaotic transport that goes in a somewhat different direction from our work here. New geometric phase-space formulations have led to important advances in the theory of transition states, especially for systems with three or more degrees of freedom. (See, for example, Ref. [22].) These developments provide new ways to identify well defined transition states, and thereby to compute transport rates or chemical reaction rates as appropriate flux integrals in phase space. Our work has some features in common with this work-most obviously our phase space perspective and more specifically our focus on a periodic 


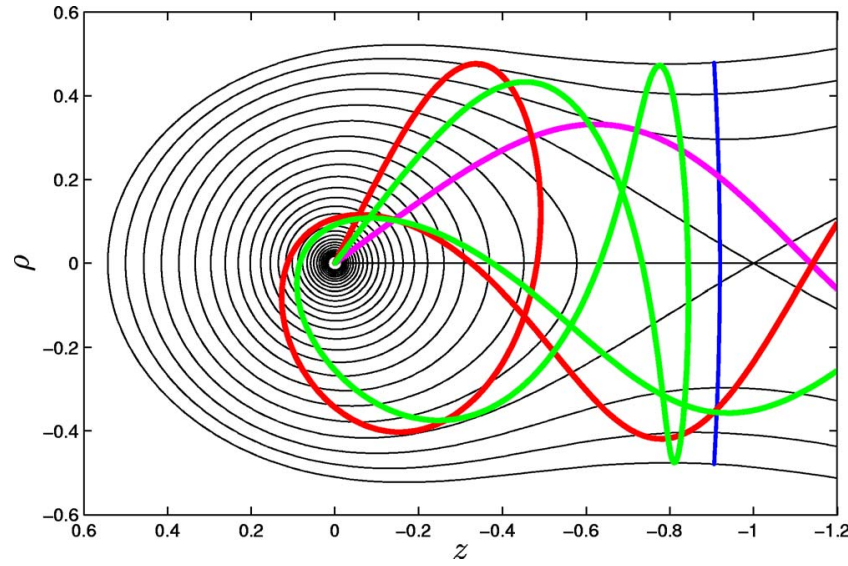

FIG. 1. (Color online) Effective potential energy contours for an electron in a hydrogen atom in parallel electric and magnetic fields, where $V(\rho, z)=\left(\rho^{2}+z^{2}\right)^{-1 / 2}+F z+B^{2} \rho^{2} / 8$, drawn in cylindrical coordinates $(\rho, z)$. The nucleus at the origin of coordinates $(\rho, z)$ $=(0,0)$ produces a deep Coulomb well, the electric field creates a saddle, and the magnetic field gives confinement in the $\rho$ direction. Near the saddle in the potential energy there is an unstable periodic orbit (the vertical line) that constitutes a dividing surface between the Coulomb well and the escape channel to the right. Electron trajectories that begin at the nucleus must pass over this dividing surface in order to escape. Three such trajectories are illustrated in the figure.

orbit dividing surface (PODS) and its associated stable and unstable manifolds. However, our goals are quite distinct. We are focused on systems with only two degrees of freedom, and we are not concerned with transition rates averaged over a microcanonical ensemble of initial conditions. Instead, we are concerned with the details of the spectrum of escape times for trajectories that begin at a single point in space at some sharply defined instant of time, and we analyze this time spectrum in terms of topological constraints placed on trajectories by homoclinic tangles.

This paper has the following outline. Section II defines the geometry of the vase cavity, and Sec. III gives an overview of the trajectories found therein, including a discussion of the escape-time plot. Section IV introduces the Poincaré return map and discusses the homoclinic tangle that underlies the escape dynamics. Section V describes the method of homotopic lobe dynamics, and Sec. VI uses this method to analyze the structure of the escape-time plot. Finally, the pulse train that is emitted from the cavity is discussed in Sec. VII.

\section{THE VASE}

We construct a two-dimensional reflecting cavity that has trajectories analogous to those of hydrogen in parallel fields (Fig. 1). In the hydrogen system, an electron may be trapped in bound trajectories which orbit the proton for all time. Many of these bound trajectories lie within a region of stability surrounding a stable periodic orbit. Outside this stable region, the electron may escape by passing over a saddle in the potential energy and into the exit channel on the right.
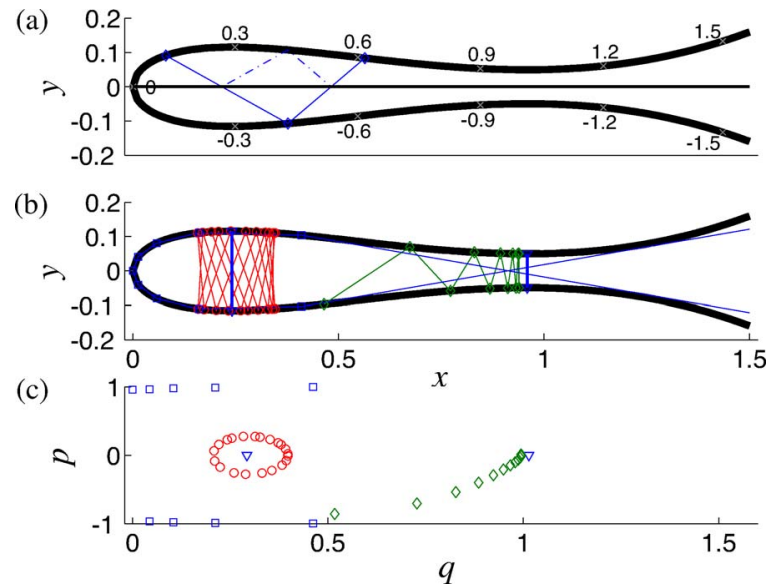

FIG. 2. (Color online) (a) A vase-shaped cavity, whose reflective boundary is defined by Eq. (1). The arc length $q$ along the boundary is recorded at several points. To simplify the surface of section, we introduce a reflective boundary at the symmetry axis $y=0$. (b) A sampling of trajectories in the vase. At the widest point of the vase body is a stable periodic orbit, with a quasiperiodic orbit nearby. At the narrowest point of the neck is an unstable periodic orbit, which forms a periodic orbit dividing surface. A trajectory is shown falling away from this periodic orbit. Finally, there is a regular scattering trajectory, or open whispering gallery mode. It starts at the lower right, outside the vase near $x=1.5, y=-0.1$. It barely misses the neck of the vase, and then hits the upper boundary almost tangentially. It continues reflecting close to the upper boundary, crosses $y=0$, and continues along the lower boundary until finally exiting at $x=1.5$. (c) Surface-of-section plot showing the same trajectories as panel (b). The two (blue) triangles near $(q, p) \approx(0.3,0)$ and $(1.02,0)$ are the intersections of the stable and unstable POs with the vase boundary. The loop of (red) circles represents the quasiperiodic trajectory shown in (b). The (green) diamonds represent the trajectory falling away from the unstable PO. The (blue) squares form the regular scattering trajectory. Its incoming reflections form the sequence of points near $p=-1$ with decreasing $q$. When the trajectory crosses $y=0, q$ becomes negative, and so we replace $(q, p)$ by $(-q,-p)$, producing an outgoing sequence of reflections near $p=1$.

The boundary between the Coulomb well and the exit channel is formed by an unstable periodic orbit near the saddle. In molecular dynamics, such a boundary is called a periodic orbit dividing surface (PODS). Some orbits approach the saddle, oscillate near the PODS, and then either pass over the PODS into the exit channel or reflect back into the Coulomb well. Electrons that cross the PODS never return to the atom.

We construct an open vase-shaped reflecting cavity with similar dynamics (Fig. 2). It has bound trajectories that bounce back and forth forever without escaping; again many of these bound trajectories lie within a region of stability surrounding a stable periodic orbit. Trajectories can only escape the cavity by passing through the bottleneck of the vase. Within the bottleneck is an unstable periodic orbit that constitutes a PODS, i.e., all escaping trajectories pass over this orbit. Again, some orbits approach the PODS, oscillate near it for a while, and then either escape from the cavity or reflect back into the interior. Past the bottleneck, the cavity flares, so that trajectories that escape are never reflected back into the cavity. 
For the boundary of the vase, we choose the function

$$
y= \pm f(x)= \pm \sqrt{x}\left[\frac{w}{2}+A(x-1)^{2}\right] .
$$

Here $w$ is a measure of the width of the vase neck, and $A$ is a measure of the curvature near the neck. In our calculations we set $w=0.1$ and $A=0.3215$. We consider a detector spanning a vertical line that is well to the right of the neck at $x$ $=1.5$. In our computations, we run trajectories from a point inside the vase until they arrive at the detector, which may be regarded as an absorbing boundary.

A shape such as this-indeed any boundary that is not convex-can create problems in the theoretical analysis. We describe the reflection of rays by recording the position $q_{n}$ along the boundary and the angle of incidence $\theta_{n}$ for successive reflections. The mapping $\left(q_{n}, \theta_{n}\right) \mapsto\left(q_{n+1}, \theta_{n+1}\right)$ will contain discontinuities if the boundary is not convex. However, in this paper, we have chosen the parameters $A$ and $w$ so that such discontinuities can only occur after the last reflection before the trajectory hits the detector. These discontinuities therefore do not invalidate our topological analysis.

We consider a burst of trajectories (light, microwaves, or particles) starting from a single initial point on the boundary of the vase and traveling in all directions into the vase. All trajectories travel with the constant speed $c=1$. This ensemble of trajectories strikes the detector in a train of pulses. To study this pulse train, we compute the escape-time plot: the time required for a trajectory launched in a given direction to reach the detector.

\section{THE ESCAPE-TIME PLOT}

There is a stable periodic orbit bouncing vertically at the widest point of the vase. Near this orbit are many quasiperiodic orbits that bounce between the upper and lower boundaries in Fig. 2(b). They move slowly back and forth in the horizontal direction, being "reflected" from the narrow tip and narrow neck of the vase, so that they remain forever in the wide part of the vase. As mentioned previously, there is an unstable periodic orbit at the narrowest point of the neck, and this orbit constitutes a PODS.

Regular scattering trajectories are also present. An easy way to construct one is to pick any point on the convex part of the boundary of the vase (a point sufficiently to the left of the neck), and start a trajectory nearly tangent to the boundary. Tracing this trajectory backwards and forwards, one obtains a regular scattering trajectory. It could be called an "open whispering gallery mode."

In addition to the bound trajectories and the regular scattering trajectories there are complex "chaotic" scattering trajectories. These trajectories may be turned back by the bottleneck many times before finally escaping, and the residence time of these trajectories within the cavity is a sensitive function of the initial conditions.

We choose an initial point on the boundary at $x=0.0466$, $y=0.0739$. (This point is selected because it makes the topological analysis as simple as possible.) In Fig. 3 we show the time to reach the detector as a function of $p=\sin \theta$, where $\theta$ is the initial launch angle of the trajectory, measured from

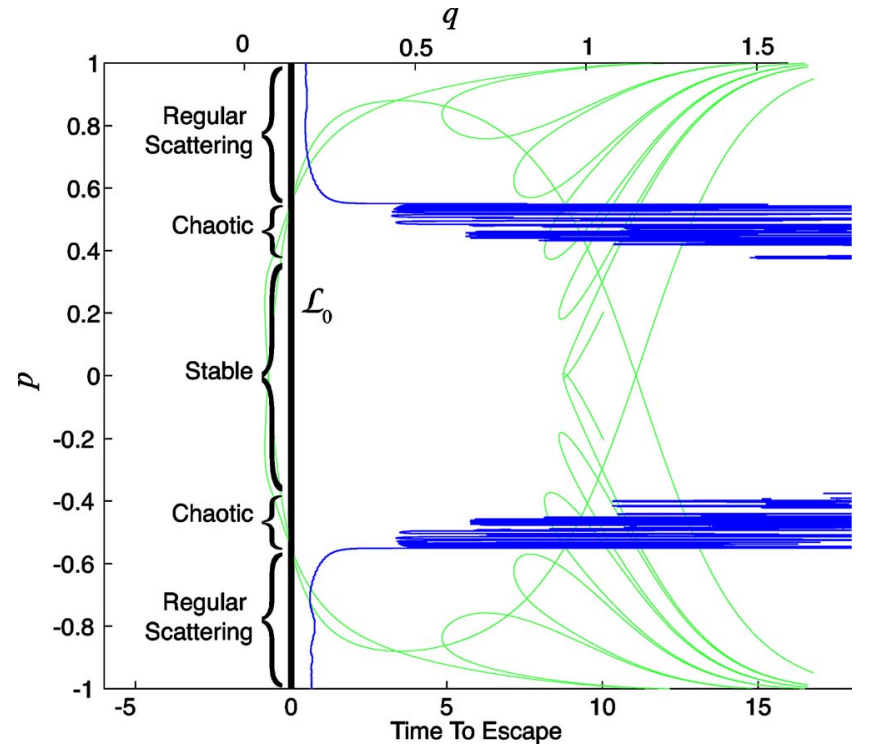

FIG. 3. (Color online) For trajectories starting at $q=0.09113$ (i.e., $x=0.0466, y=0.0739$ ), the heavy (blue) curves show the escape time (lower horizontal axis) versus the initial tangential momentum $p$ (vertical axis). When $p$ is close to zero, trajectories remain in the vase a very long time, and appear to be quasiperiodic. When $p$ is close to \pm 1 , the regular scattering trajectories escape quickly. For $|p|$ between about 0.38 and 0.55 , the escape time is a sensitive function of the initial direction. The homoclinic tangle is shown placed over the escape-time plot, with its $q$ coordinate recorded along the upper horizontal axis.

the normal. For $p$ close to zero, the initial velocity is nearly perpendicular to the boundary, and the resulting trajectories appear to be bound and quasiperiodic. For $p$ close to \pm 1 , the initial velocity is nearly tangent to the surface, and the resulting regular scattering trajectories escape quickly.

Within the two intervals of $p$ between the bound and the regular scattering trajectories, there are chaotic trajectories, where the time to escape is a sensitive function of initial conditions. The escape time in one of these intervals is shown in more detail in Fig. 4, together with two enlargements. These enlargements show repeating structure as well as variable structure at higher levels of resolution.

Our goal is to understand the structure within this escapetime plot. For this purpose, we use a Poincaré surface of section.

\section{DISCRETE TIME DYNAMICS}

\section{A. The arc-length surface of section}

We define a surface of section (SOS) in the usual way for a billiard system [Fig. 2(c)]. We assign to each point on the boundary of the vase a coordinate $q$ equal to the signed arc length along the boundary measured clockwise from the tip of the vase. Points on the upper boundary have $q>0$ and points on the lower boundary have $q<0$. The conjugate momentum $p$ is the component of velocity parallel to the boundary at the point $q$. The SOS is thus parametrized by $(q, p)$. For $|p|$ close to 1 , the trajectory is nearly grazing the surface 


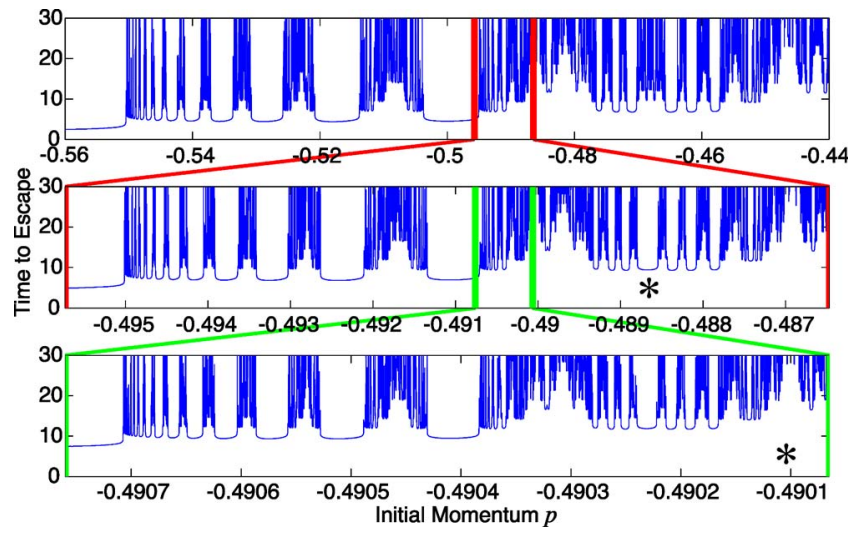

FIG. 4. (Color online) Escape time as a function of initial tangential momentum $p$. Selected regions are blown up. Repeating patterns are evident in these expanded graphs. However, we also see regions that are not repetitions of structure at larger scales. Two of these regions are marked with asterisks.

and moving in a clockwise direction; for $p$ close to 0 , the trajectory is nearly normal to the surface.

Any point $(q, p)$ specifies an initial location and direction of a ray at the boundary of the vase. Following this ray, we find a new reflection point $q^{\prime}$ where the ray next hits the boundary. The new momentum $p^{\prime}$ is the component of velocity parallel to the boundary at $q^{\prime}$. Thus, successive reflections define a map $M$ on the surface of section

$$
\left(q^{\prime}, p^{\prime}\right)=M(q, p) \text {. }
$$

We next cut the SOS in half by taking advantage of reflection symmetry. If a trajectory begins at $\left(q_{0}, p_{0}\right)$ on the upper boundary and arrives at $\left(q_{1}, p_{1}\right)$ on the lower boundary, we may instead imagine that the trajectory reflects from a flat mirror at $y=0$. [Such a trajectory is shown in Fig. 2(a).] This is equivalent to the following rule: if $q_{1}<0$, replace $\left(q_{1}, p_{1}\right)$ by $\left(-q_{1},-p_{1}\right)$. The resulting reduced $\operatorname{SOS}(q, p)$ is then defined only for $q \geqslant 0$. On the full SOS, many orbits alternate between positive and negative $q$. This includes the previously mentioned unstable orbit in the neck of the vase and the stable orbit in the body of the vase, which therefore both have period two. However on the reduced SOS, since $q$ is always positive these two orbits have period one, and thus each is a single point on the reduced SOS.

\section{B. Homoclinic tangles}

A numerically computed SOS plot is shown in Figs. 5 and 6. The stable orbit intersects the surface of section at an "O point," surrounded by rings consisting of quasiperiodic orbits. These rings bound a continent of stability. Around this continent are many smaller islands of stability. The PODS in the neck of the vase intersects the SOS at an "X point." The upper left stable manifold and the lower left unstable manifold of the $\mathrm{X}$ point contain trajectories that approach the PODS in the limit $t \rightarrow \pm \infty$. These stable and unstable manifolds intersect an infinite number of times, forming a complicated pattern called a homoclinic tangle.

Figure 7 shows a sketch of a homoclinic tangle similar to the one in Fig. 6, but which is simpler to discuss. We define

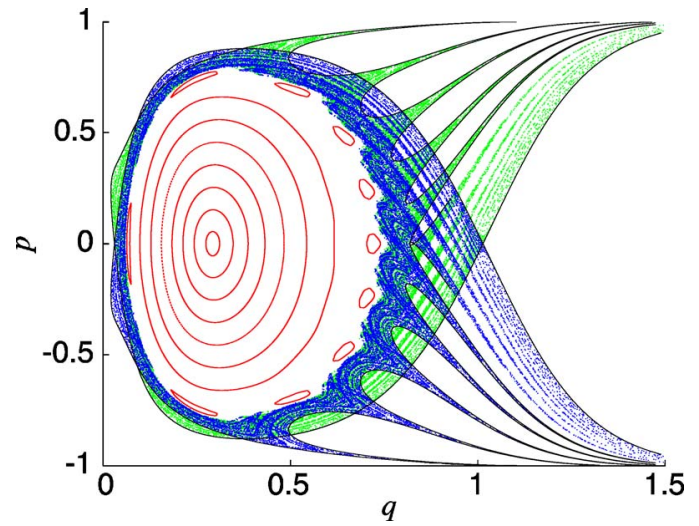

FIG. 5. (Color online) Surface of section. Concentric loops represent bound, apparently quasiperiodic trajectories. Surrounding this stable continent is a ten-island chain. All other points make up the unstable (lighter/green) and stable (darker/blue) manifolds of the unstable fixed point. The stable and unstable manifolds constitute the homoclinic tangle.

the complex as the region of the SOS bounded by the stable and unstable manifolds that connect the $\mathrm{X}$ point $\mathbf{z}_{X}$ with the intersection point $\mathbf{P}_{0}$. The point $\mathbf{P}_{0}$ maps forward to the points $\mathbf{P}_{1}, \mathbf{P}_{2}, \ldots$, and backward to $\mathbf{P}_{-1}, \mathbf{P}_{-2}, \ldots$. Between $\mathbf{P}_{0}$ and $\mathbf{P}_{1}$ is another intersection point $\mathbf{Q}_{0}$, which maps forward and backward to create the sequence of points $\mathbf{Q}_{n}$. The segments of the stable and unstable manifolds that join $\mathbf{P}_{0}$ to $\mathbf{Q}_{0}$ bound a region of the plane called the escape lobe $E_{0}$. This lobe maps backward to the escape lobes $E_{-1}, E_{-2}, \ldots$. The lobe $E_{-1}$, which is inside the complex, thus maps to the lobe

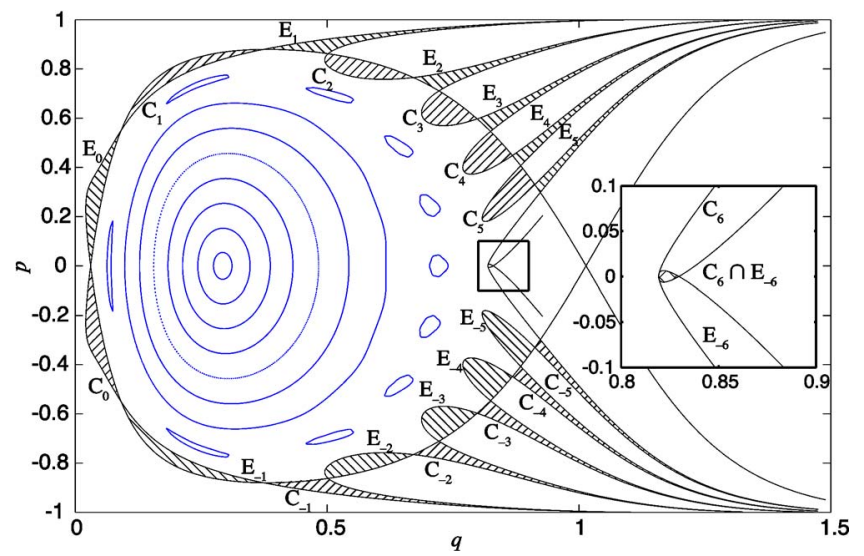

FIG. 6. (Color online) The early development of the homoclinic tangle. The $\mathrm{X}$ point near $q=1, p=0$ is the unstable PO, with its stable and unstable manifolds. One stable manifold approaches the $\mathrm{X}$ point from the lower right, and one unstable manifold recedes from it toward the upper right. These two never intersect any other manifolds. Another unstable manifold starts from the $\mathrm{X}$ point to the lower left and a stable manifold approaches the $\mathrm{X}$ point from the upper left. These two intersect near $(q=0.1, p=0)$. The interior of the region thus formed is called the complex. $C_{0}$ is a capture lobe outside the complex, which maps to $C_{1}$ inside the complex. $E_{-1}$ is an escape lobe inside the complex, which maps to $E_{0}$ outside the complex. $C_{6}$ (the sixth iterate of $C_{0}$ ) intersects $E_{-6}$ (the sixth backiterate of $E_{0}$ ), so the minimum time a scattering trajectory can spend inside the complex (i.e., the minimum delay time) is $D=11$. 


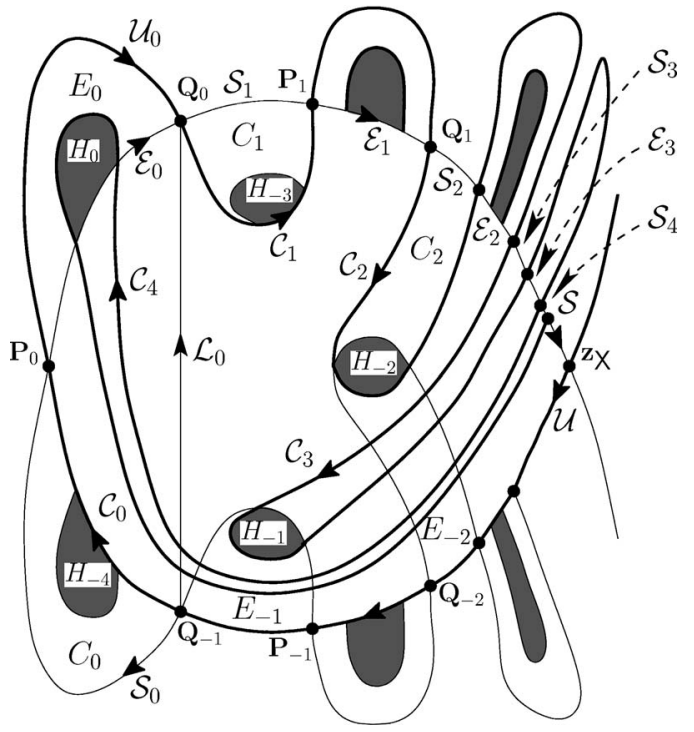

FIG. 7. Qualitative surface of section for delay time $D=3$. Points are labeled in Roman bold, curves in script, and regions of the plane in italic. $\mathbf{z}_{X}$ is the unstable fixed point, corresponding to the periodic orbit dividing surface. $\mathcal{S}$ and $\mathcal{U}$ are its stable and unstable manifolds. $\mathbf{P}_{0}$ is chosen to be the principal intersection between $\mathcal{S}$ and $\mathcal{U}$. The complex is the region of the plane bounded by the segments of $\mathcal{S}$ and $\mathcal{U}$ connecting $\mathbf{z}_{X}$ to $\mathbf{P}_{0}$. Each capture lobe, $C_{n}$, is bounded on the outside by a segment of the stable manifold $\mathcal{S}_{n}$ and on the inside by a section of the unstable manifold $\mathcal{C}_{n}$. Each escape lobe $E_{n}$ is bounded on the outside by a portion of the unstable manifold $\mathcal{U}_{n}$ and on the inside by a portion of the stable manifold $\mathcal{E}_{n}$. Capture lobe $C_{2}$ and escape lobe $E_{-2}$ overlap to form the hole $H_{-2}$. Mapping this hole backwards and forwards produces an infinite sequence of holes $H_{n}$, three of which lie inside the complex. The line of initial conditions, labeled $\mathcal{L}_{0}$, can be continuously distorted into $\mathcal{C}_{0} \mathcal{E}_{0}$ without passing through a hole, and so we have the homotopy relation $l_{0}=c_{0} e_{0}$. By scrutinizing the figure, we see that $\mathcal{C}_{4}$ can be similarly distorted into $\left(\mathcal{E}_{3}^{-1} \mathcal{C}_{3}^{-1}\right)\left(\mathcal{E}_{2}^{-1} \mathcal{C}_{2}^{-1}\right)$ $\times\left(\mathcal{E}_{1}^{-1} \mathcal{C}_{1}^{-1}\right) \mathcal{U}_{0}^{-1} \mathcal{E}_{0}\left(\mathcal{E}_{1} \mathcal{C}_{1}\right)\left(\mathcal{E}_{2} \mathcal{C}_{2}\right)\left(\mathcal{E}_{3} \mathcal{C}_{3}\right) \mathcal{S}_{4}$, and so we arrive at Eq. (5).

$E_{0}$, which is outside the complex. We say that a point has escaped from the complex when it arrives in $E_{0}$. Any point that lands in $E_{0}$ must map to successive escape lobes $E_{n}$, and therefore escape from the cavity; such points may take a number of additional bounces before arriving at the detector, but calculations show that they never reenter the cavity.

The segments of the stable and unstable manifolds that join $\mathbf{Q}_{-1}$ to $\mathbf{P}_{0}$ bound the capture lobe $C_{0}$, which maps forward to the lobes $C_{1}, C_{2}, \ldots$. The lobe $C_{0}$, which is outside the complex, maps to the lobe $C_{1}$, which is inside the complex, and we say that points in $C_{1}$ have just been captured into the complex. The lobes $C_{0}$ and $E_{-1}$ are sometimes called a "turnstile [23]."

Notice in Fig. 7 that the lobes $C_{2}$ and $E_{-2}$ intersect in the region $H_{-2}$. Mapping this intersection forward twice, we see that $C_{4}$ and $E_{0}$ also intersect. We define the minimum delay time $D$ to be the smallest $n$ such that $C_{n+1}$ intersects $E_{0}$; that is, $D$ is the smallest number of iterates that a scattering trajectory may spend within the complex. In Fig. 7, $D=3$. The lobe $C_{0}$ (outside) maps to $C_{1}$ (inside) which maps to $C_{2}$ (inside); some of the points in $C_{2}$ are also in $E_{-2}$; they map to

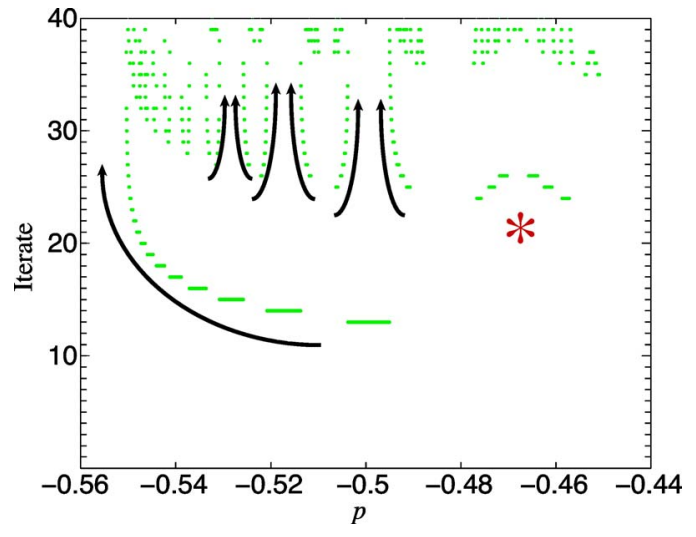

FIG. 8. (Color online) Discrete-escape-time plot. Plotting the iterate at escape (arrival in $E_{0}$ ) versus $p$, the icicles in Fig. 4 are rectified into escape segments. Many escape segments form regular sequences which we call epistrophes. In the range of $p$ shown here, the minimal set of escape segments begins at $i=13$, and the first epistrophe converges to the left. Each segment of this epistrophe spawns a new epistrophe beginning twelve iterates later. Then each segment in each of those epistrophes must spawn a new epistrophe beginning twelve iterates later. In contrast, the cluster of escape segments marked by an asterisk is not part of the minimal set, and this cluster cannot be predicted by the topological methods used here.

$E_{-1}$ (inside) and then to $E_{0}$ (outside); therefore such trajectories spend three iterates inside the complex, and no trajectory coming from outside can spend fewer iterates inside. Examining the surface of section for the vase in Fig. 6, there is an intersection between $C_{6}$ and $E_{-6}$ which implies $D=11$.

\section{The discrete-escape-time plot}

The ensemble of initial conditions used to compute the escape-time plots (Figs. 3 and 4 ) forms a line $\mathcal{L}_{0}$ in the surface of section. This line of initial conditions appears as the vertical line in Fig. 3, with $q=0.09113$ and $p$ ranging between \pm 1 . The map $M$ defines a discrete-escape-time plot (Fig. 8), in which we record the iterate at which a point arrives in $E_{0}$ as a function of $p$ along $\mathcal{L}_{0}$. This discreteescape-time plot rectifies the continuous-escape-time plot (Fig. 4). Each icicle in Fig. 4 is straightened into a constant escape segment. Each escape segment spans an open interval of $\mathcal{L}_{0}$ in which all points arrive in $E_{0}$ on the same iterate.

Many of these escape segments form orderly sequences. Several such sequences are marked by arrows in Fig. 8. The long left pointing arrow marks a sequence that converges upon the boundary of the complex, where chaotic scattering trajectories change to regular scattering trajectories. The other arrows mark additional sequences which converge upon the endpoints of earlier escape segments. We call each such regular sequence of escape segments an epistrophe.

The "epistrophe theorem" proved in Ref. [1] asserts that (1) such a sequence of escape segments converges upon every endpoint of every escape segment and (2) the asymptotic tails of such sequences are self-similar and are all similar to each other. The epistrophe theorem therefore implies that there must be regular structure-within-structure at all levels 
of resolution in the escape-time plot. On the other hand, the epistrophe theorem says nothing about how epistrophes begin, nor does it assert that all escape segments lie in well organized epistrophes. (Some segments that are not part of epistrophes are marked with an asterisk in Fig. 8.)

Homotopic lobe dynamics is a topological method for explaining, or predicting, the regular sequences of escape segments shown in Fig. 8. The next section describes this method. Further details are given in Refs. [2,11].

\section{HOMOTOPIC LOBE DYNAMICS}

As explained above, for the tangle sketched in Fig. 7, $C_{2}$ intersects $E_{-2}$, and so the minimum delay time $D$ is equal to three. We consider the intersection $H_{-2}=C_{2} \cap E_{-2}$ to be a "hole" in the SOS. We consider all its forward and backward iterates $H_{n}$ to be holes as well. Each $H_{n}$ lies in the escape lobe $E_{n}$ and in the capture lobe $C_{D+n+1}$.

Now we label the segments forming the boundaries of the escape and capture lobes. We label the outer boundary of the capture lobe $C_{n}$ by $\mathcal{S}_{n}$, since it lies on the stable manifold. We label the inner boundary of $C_{n}$ by $\mathcal{C}_{n}$. Similarly, we label the outer boundary of the escape lobe $E_{n}$ by $\mathcal{U}_{n}$, since it lies on the unstable manifold. We label the inner boundary of $E_{n}$ by $\mathcal{E}_{n}$. Each of these boundaries is really a directed curve or path; they inherit their direction from the directions of the stable and unstable manifolds [24].

\section{A. Path classes}

We now define families of equivalent paths, or "path classes." The path class $c_{n}$ is the family of all paths obtained by distorting the curve $\mathcal{C}_{n}$ without crossing any hole $H_{k}$ or moving its endpoints. Likewise $s_{n}, e_{n}$, and $u_{n}$ are respectively the families of paths obtained by distorting the curves $\mathcal{S}_{n}, \mathcal{E}_{n}$, and $\mathcal{U}_{n}$ without crossing any hole or moving endpoints. Similarly, $l_{0}$ is the path class obtained by distorting that part of the curve $\mathcal{L}_{0}$ joining $\mathbf{Q}_{-1}$ to $\mathbf{Q}_{0}$, without intersecting any hole. For example, in Fig. 7 we see that the path $\mathcal{L}_{0}$ can be distorted into the combination of directed curves $\mathcal{C}_{0}$ followed by $\mathcal{E}_{0}$. We say that path $\mathcal{L}_{0}$ is homotopic to path $\mathcal{C}_{0}$ followed by path $\mathcal{E}_{0}$; i.e., the path class $l_{0}$ is the same as the path class $c_{0}$ followed by $e_{0}$ :

$$
l_{0}=c_{0} e_{0} .
$$

More generally, path classes can be combined by multiplication and inversion. Two path classes $a_{1}$ and $a_{2}$ may be multiplied to form a new path class $a_{1} a_{2}$ if the final point of $a_{1}$ is the initial point of $a_{2}$. Any path in $a_{1} a_{2}$ is homotopic to any path in $a_{1}$ followed by any path in $a_{2}$. We define the inverse $a^{-1}$ of a path class $a$ as the family of all paths in $a$ traversed backwards. The product of any path class with its inverse is the identity path class 1 , the set of all paths beginning and ending at the same point without encircling any holes, i.e., $a a^{-1}=1$. Similarly, $a 1=1 a=a$. These properties define a mathematical group, except that multiplication must be between adjacent paths. (To combine two paths $a$ and $b$, the final point of $a$ must equal the initial point of $b$.) Hence, the path classes $s_{n}, u_{n}, e_{n}$, and $c_{n}$ generate what mathemati- cians call a "groupoid" start from here. This groupoid consists of the set of all path classes that begin and end at any of the homoclinic points $\mathbf{P}_{i}$ and $\mathbf{Q}_{j}$ and encircle any collection of holes $H_{k}$ any number of times, in any direction and in any order. The map $M$, acting on these path classes, simply shifts their indices

$$
\begin{aligned}
& M\left(s_{n}\right)=s_{n+1}, \\
& M\left(u_{n}\right)=u_{n+1}, \\
& M\left(e_{n}\right)=e_{n+1}, \\
& M\left(c_{n}\right)=c_{n+1} .
\end{aligned}
$$

\section{B. The basis of path classes}

The elements $s_{n}, u_{n}, c_{n}, e_{n}$ generate the groupoid but they are not a minimal generating set, i.e., they are not all required to form a basis of path classes. As shown in Ref. [2], a basis can be formed by including only $s_{n}(-\infty<n<\infty)$, $u_{n}(-\infty<n<\infty), c_{n}(-\infty<n \leqslant D)$, and $e_{n}(0 \leqslant n<\infty)$.

As already stated, the line of initial conditions we chose is homotopic to $c_{0} e_{0}$. The Poincaré map $M$ acts on the decomposed path class $l_{0}=e_{0} c_{0}$ to advance the indices of each basis factor in turn, e.g., $l_{1}=M\left(l_{0}\right)=M\left(e_{0} c_{0}\right)=e_{1} c_{1}$. The forward iterates of $l_{0}$ will automatically be expressed in the basis until we reach $M\left(l_{D}\right)=e_{D+1} c_{D+1}$. The factor $c_{D+1}$ is not in the basis, but it can be decomposed into basis elements through a lengthy "unwinding" process, which can be extracted from Fig. 7 for the case $D=3$ :

$$
\begin{aligned}
c_{4}= & \left(e_{3}^{-1} c_{3}^{-1}\right)\left(e_{2}^{-1} c_{2}^{-1}\right)\left(e_{1}^{-1} c_{1}^{-1}\right) u_{0}^{-1} \\
& \times e_{0}\left(e_{1} c_{1}\right)\left(e_{2} c_{2}\right)\left(e_{3} c_{3}\right) s_{4} .
\end{aligned}
$$

This is explained in greater detail in Ref. [2], in which the following formula for arbitrary $D$ is obtained:

$$
M\left(c_{D}\right)=F^{-1} u_{0}^{-1} e_{0} F s_{D+1},
$$

where

$$
F=\left(c_{1} e_{1}\right)\left(c_{2} e_{2}\right) \cdots\left(c_{D} e_{D}\right) .
$$

The product $F$ itself can be shown to map forward as

$$
M(F)=e_{1}^{-1} c_{1}^{-1} u_{0}^{-1} e_{0} F s_{D+1} e_{D+1} .
$$

\section{Symbolic representation of escape}

The above algebra generates a symbolic basis expansion for all forward iterates $l_{n}=M^{n}\left(l_{0}\right)$ of the path class for the line of initial conditions. Moreover, the path class $l_{n}$ passes through the escape lobe $E_{0}$ once for every occurrence of $u_{0}$ or $u_{0}^{-1}$ in its basis expansion. This is clear from a plot of the tangle. The segment $\mathcal{U}_{0}$ bounds $E_{0}$ on the outside. Therefore, any segment of $\mathcal{L}_{n}$ that has mapped from inside the complex to a curve in class $u_{0}$ must therefore cross $E_{0}$, and thereby generate a segment that escapes on the $n$th iterate. This is true for either $u_{0}$ or $u_{0}^{-1}$, which determines whether $\mathcal{L}_{n}$ travels 
clockwise or counterclockwise around the hole $H_{0}$. Thus, this algebraic representation yields a topologically forced set of intersections of $\mathcal{L}_{n}$ with $E_{0}$; i.e., it gives a minimal required set of escape segments.

The algorithm for generating this minimal set is summarized by the following.

(1) Decompose $l_{0}$ in the basis.

(2) Iterate this decomposition for $l_{0}$ forward $N$ times, applying Eqs. (4), (6), and (8) [or alternatively Eqs. (9) below]. Cancel any products $a a^{-1}$.

(3) Each $u_{n}$ or $u_{n}^{-1}$ factor $(n \geqslant 0)$ in the decomposition of $l_{N}$ corresponds to a segment that escapes in $N-n$ iterates.

(4) The relative positions of the $u_{n}$ factors in the expansion of $l_{N}$ are the same as the relative positions of their corresponding escape segments along $\mathcal{L}_{0}$.

This algorithm can be simplified further. All segments $e_{n}(n \geqslant 0)$ and $s_{n}(-\infty<n<\infty)$ can be eliminated from the algebraic process, because they never spawn any $u_{0}$ segments, and because their omission does not lead to any false cancellation of factors. Thus, the dynamical equations needed in step 2 of the algorithm reduce to

$$
\begin{aligned}
& M\left(u_{n}\right)=u_{n+1}, \quad-\infty<n<\infty, \\
& M\left(c_{n}\right)=c_{n+1}, \quad n<D, \\
& M\left(c_{D}\right)=F^{-1} u_{0}^{-1} F, \\
& M(F)=c_{1}^{-1} u_{0}^{-1} F .
\end{aligned}
$$

In Ref. [2] it is shown that at sufficiently large $N$, the algorithm implies the following two rules.

Epistrophe continuation rule. Every segment in the minimal set that escapes at $N-1$ iterates has beside it a segment that escapes at $N$ iterates. This is the origin of epistrophes in the escape-time plot.

Epistrophe start rule. Every segment in the minimal set that escapes at $N-\Delta$ iterates $(\Delta=D+1)$ spawns on both sides a segment that escapes at $N$ iterates. Each of these segments begins an epistrophe that converges toward the spawning segment.

The algorithm and the two rules predict only a minimal topologically forced set of escape segments. They do not predict or explain any others. In fact other escape segments typically do occur, and they are caused by additional twisting and mangling of $\mathcal{L}_{0}$ at higher iterates of the map. The description of all escape segments would require not only the minimum delay time $D$, but also an infinity of other parameters describing the topology of the tangle.

\section{ANALYSIS OF THE ESCAPE-TIME PLOT}

We now analyze the escape-time plot for the vase (Figs. 8 and 9). As noted in Sec. IV B, for the parameters considered here, the intersection between $C_{6}$ and $E_{-6}$ yields $D=11$ (Fig. $6)$. We choose the location of the source of particles to be at $q=0.09113$, so that the resulting line of initial conditions $\mathcal{L}_{0}$ passes through the points $\mathbf{Q}_{0}$ and $\mathbf{Q}_{-1}$ on the SOS. Consequently, $l_{0}=c_{0} e_{0}$ as in Eq. (3) and Fig. 7. (For other lines of initial conditions, see Ref. [2].)

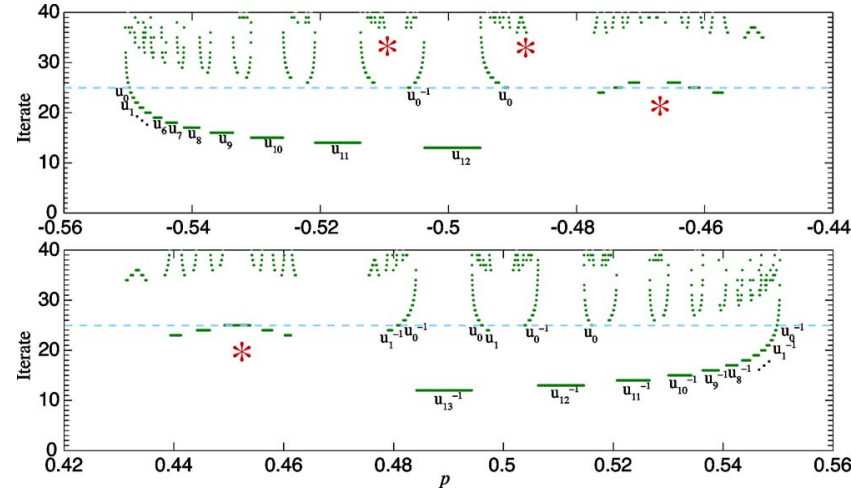

FIG. 9. (Color online) Escape segments in the minimal set are labeled according to their factors in $l_{25}$. In expression (11), each $u_{0}$ represents an escape segment that occurs on the 25th iterate, and each $u_{n}$ for $n>0$ represents a segment that escapes on iterate $i=25-n$. Thus the segments in the epistrophe that begins near $p=-0.5$ at $i=13$ are labeled $u_{12}, u_{11}, \ldots, u_{0}$. On either side of the first $u_{12}$ in expression (11) is a $u_{0}$, and these represent the first segments of the epistrophes that converge on either endpoint of the segment labeled $u_{12}$. Similarly, $u_{13}$ is a segment near $p=+0.49$ that escapes on iterate $i=12$. On either side of $u_{13}$ in expression (11) is both a $u_{0}$ and a $u_{1}$, which are the first two segments of the epistrophes that converge upon $u_{13}$.

We apply the preceding algorithm, ignoring all $e_{n}$ and $s_{n}$ segments, to obtain

$$
\begin{aligned}
l_{0} & =c_{0}, \\
l_{1} & =c_{1}, \\
l_{2} & =c_{2}, \\
& \vdots \\
l_{11} & =c_{11}, \\
l_{12} & =F^{-1} u_{0}^{-1} F, \\
l_{13} & =F^{-1} u_{0}^{-1} c_{1} u_{1}^{-1} c_{1}^{-1} u_{0}^{-1} F .
\end{aligned}
$$

The first $u_{0}$ factor occurs at iterate 12 , signifying one escape segment on that iterate. The next iterate $l_{13}$ has a $u_{1}^{-1}$ and two $u_{0}$ 's, corresponding to the one segment already found on iterate 12 and two new segments on iterate 13 . Continuing this iteration, one may verify by hand that $l_{25}$ contains the following $u$ factors in order:

$$
\begin{gathered}
u_{0}, u_{1}, \ldots, u_{11}, u_{0}^{-1}, u_{12}, u_{0} ; \\
u_{1}^{-1}, u_{0}^{-1}, u_{13}^{-1}, u_{0}, u_{1}, u_{0}^{-1}, u_{12}^{-1}, u_{0}, u_{11}^{-1}, u_{10}^{-1}, \ldots, u_{0}^{-1} .
\end{gathered}
$$

As stated earlier, each $u_{n}$ in this list is a segment that arrived in the escape lobe $E_{0}$ on the $i=25-n$ iterate of the map. This algorithm predicts many of the escape segments seen in Fig. 9. For example, $u_{0}, u_{1}, \cdots, u_{11}$ together with $u_{12}$ are part of an epistrophe which begins on the thirteenth iterate. On 
either side of $u_{12}$ is a $u_{0}^{-1}$ and a $u_{0}$ segment, which escape at $i=25$. These $u_{0}^{-1}$ and $u_{0}$ segments are the beginnings of two new epistrophes converging upon $u_{12}$. This agrees with the epistrophe start rule, which states that an epistrophe must begin $\Delta=12$ iterates after the segment upon which it converges.

Comparing expression (11) to Fig. 9, we find that all segments predicted by the topological algorithm are present in the numerical computation. All segments before the semicolon in expression (11) occur in the upper part of Fig. 9, and all segments after the semicolon occur in the lower part of Fig. 9.

On the other hand, some escape segments shown in Fig. 9 are not part of the minimal set, and cannot be predicted from the present topological arguments. For example, every escape segment in the interval $-0.48<p<+0.47$ is not part of the minimal set. We see two large groups of such escape segments marked by asterisks: a set of six near $p=-0.47$ and another set near $p=+0.45$. Note that they are the largest segments in the range $i=23, \ldots, 26$.

The "epistrophe theorem" proved in Ref. [1] asserts that an epistrophe must converge upon each end of every escape segment. However, except in the minimal set, we cannot predict when these epistrophes begin. Scrutiny of the upper parts of Fig. 9 indicates that even in the unpredicted parts of the figure, epistrophes often begin 12 iterates after the segment upon which they converge.

We have used the word "strophe" to refer to segments that are not part of the minimal set, i.e., are not predicted by this topological analysis. The present methods permit the existence of strophes, but they tell us nothing about them-they do not tell us whether the strophes form repeating patterns or what patterns might be present. We have recently succeeded in generalizing the method of homotopic lobe dynamics to account for such additional strophe structures [11]. The application and development of this new method is work in progress.

\section{THE PULSE TRAIN}

For definiteness, we take the vase to be a microwave cavity, with one unit of length equal to one meter and one unit of time equal to $3.3 \mathrm{~ns}$. We consider a pulse of radiation from a source at $x=0.0466, y=0.0739$ with a uniform distribution in $p=\sin \theta$, where $\theta$ is the angle of the ray from the normal. We numerically compute the rate at which microwaves strike the detector at $x=1.5$ as a function of time. We then convolve this time spectrum with a Gaussian of width $0.1 \mathrm{~ns}$. This width is intended to model the initial duration of the pulse, plus cavity dispersion (if any), plus measurement uncertainty in the detector.

The resulting time spectrum is shown in Fig. 10. Near $t$ $\approx 2$ is a large "prompt" pulse consisting of trajectories that begin on the two intervals of $\mathcal{L}_{0}$ outside the complex. These intervals are labeled "regular scattering" in Fig. 3. They include whispering gallery trajectories which run around the convex part of the boundary of the vase. In addition, they include trajectories that escape without any bounces from the boundary, as well as trajectories that may have a large num-

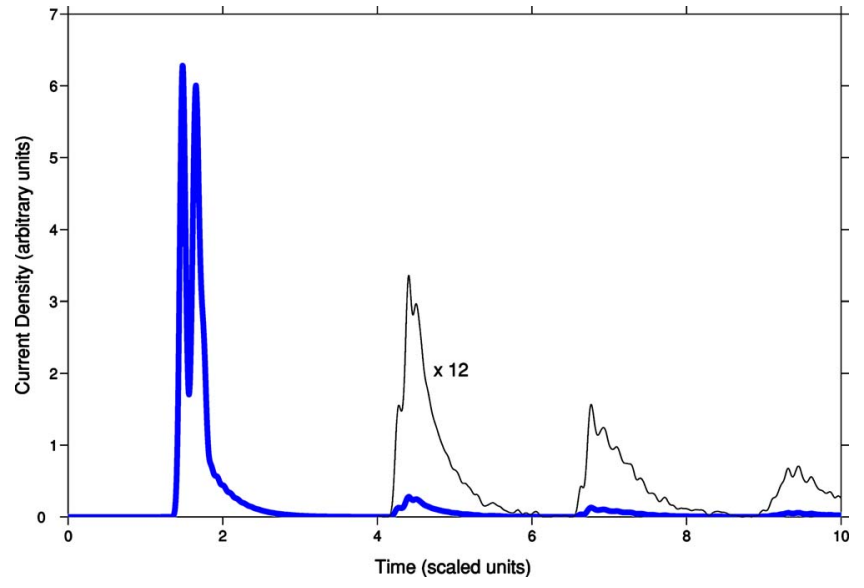

FIG. 10. (Color online) The current striking the detector at the vertical line $x=1.5$, plotted as a function of time after the initial burst of radiation. For clarity, the pulses after time 5 are reproduced, expanded by a factor of 12 .

ber of bounces but pass through the neck of the vase without being turned back.

Near $t \approx 5$ units is a pulse arising from the epistrophes that begin at iterates 12 and 13 . The trajectories approach the neck but are turned back, and then escape when they approach the second time. Near $t \approx 7$ units is a pulse associated with the strophes and epistrophes near iterate 23 . The corresponding trajectories have been turned back twice by the neck of the vase before escaping on their third approach.

For the parameters chosen, pulses arising from individual escape segments within an epistrophe are not fully resolved, but rather overlap to form the large pulses in Fig. 10. This contrasts with the hydrogen computations in which some pulses from individual escape segments were resolved $[3,4]$.

\section{CONCLUSIONS AND FUTURE WORK}

The vase was conceived as a simple and experimentally accessible system having trajectories that are analogous to those that occur in the ionization of hydrogen in parallel electric and magnetic fields. The most important aspect of the dynamics is the presence of a periodic orbit dividing surface and its associated homoclinic tangle. Unstable periodic orbits and their homoclinic or heteroclinic tangles occur generally in the theory of chaotic transport and in the modern theory of transition states [22]. The time required for a particle to escape from such a system is a sensitive function of initial conditions, and the escape-time plot shows fractal structure. We have computed the escape-time plot for the vase, and we have used the topological methods developed in Ref. [2] (homotopic lobe dynamics) to analyze and interpret the fractal structure of this plot.

There is an alternative way to observe the fractal character of the escape dynamics. Instead of using a burst of photons and measuring the time spectrum, one could use a fixedfrequency continuous source and a small (pointlike) detector to measure interferences. Each trajectory going from source to detector carries an associated wave, and the phase of this 
wave is proportional to the length of the path. Varying the frequency of the source, the signal at the detector would be a superposition of sinusoidal oscillations, and Fourier transformation would recover the time spectrum of orbits from the source to the detector. Calculations of this type are now being carried out for various vase-shaped enclosures.

\section{ACKNOWLEDGMENTS}

This research was supported by the National Science Foundation through Grant Nos. 0139167 and 0140329. We thank Melissa Commisso for preliminary calculations that helped get this project started, and we thank Jaison Novick for Fig. 10.
[1] K. A. Mitchell, J. P. Handley, B. Tighe, S. K. Knudson, and J. B. Delos, Chaos 13, 880 (2003).

[2] K. A. Mitchell, J. P. Handley, S. K. Knudson, and J. B. Delos, Chaos 13, 892 (2003).

[3] K. A. Mitchell, J. P. Handley, B. Tighe, A. Flower, and J. B. Delos, Phys. Rev. Lett. 92, 073001 (2004).

[4] K. A. Mitchell, J. P. Handley, B. Tighe, A. Flower, and J. B. Delos, Phys. Rev. A 70, 043407 (2004).

[5] This work is based in part on a thesis submitted by PH for the degree of Bachelor of Science at the College of William and Mary, 2004.

[6] C. C. Rankin and William H. Miller, J. Chem. Phys. 55, 3150 (1971).

[7] M. J. Davis and S. K. Gray, J. Chem. Phys. 84, 5389 (1986); M. J. Davis, ibid. 83, 1016 (1985).

[8] A. Tiyapan and Charles Jaffé, J. Chem. Phys. 99, 2765 (1993).

[9] P. Gaspard, Chaos, Scattering and Statistical Mechanics (Cambridge University Press, Cambridge, 1998).

[10] Preliminary studies of this system were carried out by Melissa Commisso, REU project report, College of William and Mary, 2003 (unpublished).

[11] K. A. Mitchell and J. B. Delos (unpublished).

[12] B. Rückerl and C. Jung, J. Phys. A 27, 55 (1994).

[13] B. Rückerl and C. Jung, J. Phys. A 27, 6741 (1994).

[14] C. Lipp and C. Jung, J. Phys. A 28, 6887 (1995).

[15] C. Jung, C. Lipp, and T. H. Seligman, Ann. Phys. (N.Y.) 275, 151 (1999).

[16] C. Jung and A. Emmanouilidou, Chaos 15, 023101 (2005).

[17] R. W. Easton, Trans. Am. Math. Soc. 294, 719 (1986).
[18] R. W. Easton, Geometric Methods for Discrete Dynamical Systems (Oxford, New York, 1998).

[19] V. Rom-Kedar, Physica D 43, 229 (1990).

[20] V. Rom-Kedar, Nonlinearity 7, 441 (1994).

[21] P. Collins, in Geometry and Topology in Dynamics, edited by M. Barge and K. Kuperberg (American Mathematical Society, Providence, 1999); Int. J. Bifurcation Chaos Appl. Sci. Eng. 12, 605 (2002); Dyn. Syst. 19, 1 (2004); 20, 369 (2005); Exp. Math. 14, 75 (2005); P. Collins and B. Krauskopf, Phys. Rev. E 66, 056201 (2002).

[22] B. Eckhardt, J. Phys. A 28, 3469 (1995); W. S. Koon, M. W. Lo, J. E. Marsden, and S. D. Ross, Chaos 10, 427 (2000); T. Komatsuzaki and R. S. Berry, Proc. Natl. Acad. Sci. U.S.A. 98, 7666 (2001); C. Jaffé, S. D. Ross, M. W. Lo, J. Marsden, D. Farrelly, and T. Uzer, Phys. Rev. Lett. 89, 011101 (2002); T. Uzer, C. Jaffé, J. Palacián, P. Yanguas, and S. Wiggins, Nonlinearity 15, 957 (2002); H. Waalkens and S. Wiggins, J. Phys. A 37, L435 (2004); H. Waalkens, A. Burbanks, and S. Wiggins, ibid. 37, L257 (2004); H. Waalkens, A. Burbanks, and S. Wiggins, ibid. 38, L759 (2005); H. Waalkens, A. Burbanks, and S. Wiggins, Phys. Rev. Lett. 95, 084301 (2005); F. Gabern, W. S. Koon, J. E. Marsden, and S. D. Ross, Physica D 211, 391 (2005).

[23] R. S. MacKay, J. D. Meiss, and I. C. Percival, Physica D 13, 55 (1984); S. Wiggins, Chaotic Transport in Dynamical Systems (Springer-Verlag, New York, 1992).

[24] In differential geometry a path is a parametrized directed curve. This distinction is of little importance in our presentation. 